\author{
Series A
}

\author{
I. MATHEMATICA
}

337

\title{
FUNDAMENTAL POLYGONS OF FUCHSIAN AND FUCHSOID GROUPS
}


Communicated 13 September 1963 by P. J. Myrberg and Olli Lehto

HELSINKI 1964 


\section{Fundamental Polygons of Fuchsian and Fuchsoid Groups ${ }^{1}$ )}

1. Although the theory of Fuchsian and Fuchsoid groups has been considered for many years, certain aspects concerning the properties of fundamental polygons merit renewed examination. Let $T$ denote a group of conformal automorphisms of $\Delta=\{|z|<1\}$ onto itself which is properly discontinuous (at each point of $\Delta$ ). Let $\Theta$ denote a group of Möbius transformations acting on the extended plane, mapping $\Delta$ onto itself, and properly discontinuous at each point of $\Delta$. If $\Theta$ is not properly discontinuous at any point of $\mathrm{C}=\{|z|=1\}, \Theta$ is said to be of the first kind. Otherwise, $\Theta$ is said to be of the second kind. Given a group $\Gamma$, the set of Möbius transformations whose restrictions to $\Delta$ are the members of $\Gamma$ constitutes a group $\Theta$, which we shall denote by $\Theta(\Gamma)$. Similarly, we shall denote the set of the restrictions of the members of a group $\Theta$ to $\Delta$, which constitutes a group $\Gamma$, by $\Gamma(\Theta)$.

An important result of C. L. Siegel $[9,10]$ states: if a Poincaré fundamental polygon of a group $\Gamma$ has finite hyperbolic area, then it has a finite number of sides, none of which is on $C$, and $\Theta(\Gamma)$ is of the first kind.

It is natural to inquire whether Siegel's theorem has a partial counterpart for $\Theta$ of the second kind. When Siegel's theorem is reformulated in terms of metrics of constant negative curvature associated with the quotient map defined by $\Gamma$, a natural counterpart of Siegel's theorem for $\Theta$ of the second kind is valid. It is one of our objects to establish extensions of Siegel's theorem and this result to the case of arbitrary non-euclidean convex fundamental polygons [Th. 4.2, Th. 6.1].

With the aid of these theorems we shall obtain the following theorem.

Theorem 1.1: Each non-euclidean convex fundamental polygon of a finitely generated $\Theta$ has a finite number of sides. If some non-euclidean convex fundamental polygon has a finite number of sides, then $\Theta$ is finitely generated.

We recall that in the sense of the terminology of [2] a group $\Theta$ is termed Fuchsian provided that some Poincaré normal fundamental polygon of $\Theta$ has a finite number of sides and otherwise is termed Fuchsoid. It is a consequence of part of the arguments leading to Theorem 1.1 that if some

$\left.{ }^{1}\right)$ Work on this paper was carried out during the tenure of National Science Foundation Grant NSF G 25227. 
non-euclidean convex fundamental polygon of $\Theta$, in particular a Poincaré fundamental polygon, has a finite number of sides, then so does every non-euclidean convex fundamental polygon of $\Theta$ [cf. $\S 7]$.

Our developments will be based on a free use of Riemann surface theory and the geometric reasoning employed by Siegel. In particular, we shall focus attention on the map that carries a point into its orbit with respect to $\Theta$.

In $\S 12$ we shall make a systematic study of the relation between the parabolic members of an arbitrary $\Theta$ and the cusps of an associated noneuclidean convex fundamental polygon. The results are, it is to be noted, free of finiteness conditions. The work of $\S 12$ is based on our paper [5].

2. It will be convenient to recall some basic facts concerning properly discontinuous groups of Möbius transformations. Suppose that $M$ is a group of Möbius transformations. By the orbit of a point $z$ of the extended plane with respect to $M$, denoted $\varphi(z)$, is meant the set $\{\tau(z) \mid \tau \in M\}$. Given $O$, a non-empty open subset of the extended plane, we introduce $v(z, O)$, the number of distinct $\tau \in M$ such that $\tau(z) \in O$. Clearly, $z \rightarrow v(z, O)$ is a lower semi-continuous function in the extended plane. We say that $M$ is properly discontinuous at a provided that for some $O$ containing $a$,

$$
\sup _{z \in O} v(z, O)<+\infty \text {. }
$$

The set of points at which $M$ is properly discontinuous is open and is mapped univalently onto itself by each $\tau \in M$. We suppose that the set in question is not empty. Let $\Omega$ denote a component. We introduce

$$
F(M, \Omega)=\{\varphi(z) \mid z \in \Omega\} .
$$

If we endow $F(M, \Omega)$ with the weakest topology rendering

$$
\varphi: z \rightarrow \varphi(z), z \in \Omega,
$$

an open mapping, then $F(M, \Omega)$ becomes a 2-dimensional manifold. Further, $F(M, \Omega)$ may be endowed with a conformal structure rendering $\varphi$ a conformal map of $\Omega$ onto $F(M, \Omega)$. Indeed, $F(M, \Omega)$ may be endowed with a Riemann surface structure that renders $\varphi$ conformal in essentially one way. Given $a \in \Omega$, the set

$$
\left\{\sup _{z \in O} v(z, O) \mid a \in O\right\}
$$

has a finite minimum, which is, in fact, equal to the number of distinct members of $M$ having $a$ as a fixed point and also is equal to $n(a ; \varphi)$, the multiplicity of $\varphi$ at $a$. Since $\varphi$ is automorphic with respect to $M$, 
it follows that $n(a ; \varphi)=n(b ; \varphi)$, if $b \in \varphi(a) \cap \Omega$. Thus we see that if $q \in F(M, \Omega)$, then the multiplicity of $\varphi$ is the same at each point ${ }^{1}$ of $\varphi^{-1}(\{q\})$. We denote the common value by $\partial(q)$. The function $\partial: q \rightarrow \partial(q)$, $q \in F(M, \Omega)$, has the property that the set $\{\partial(q)>1\}$ clusters at no point of $F(M, \Omega)$. We term $\partial$ the ramification index of $\varphi$.

A case of particular interest is that where each $\tau \in M$ maps $\Delta$ onto itself. In this case, if the set of points where $M$ is properly discontinuous is not empty, then either it has two components, $\Delta$ and ext $\Delta$, or else it is connected and contains $\Delta \mathrm{U}$ ext $\Delta$. The groups in question are the $\Theta$ of $\S 1$.

Poincaré's normal fundamental polygon. Given points $a, b \in \Delta$, we denote the hyperbolic distance between $a$ and $b$ by $[a, b]$. By the Poincaré normal fundamental polygon, $\Pi(a)$, of a group $\Theta$ relative to $a \in \Delta, n(a ; \varphi)=1$, is meant simply the set of $z \in \Delta$ satisfying

$$
[z, a] \leq[z, \tau(a)], \quad \tau \in \Theta
$$

(The definition of a Poincaré normal fundamental polygon of a group $\Gamma$ is the same with $\Gamma$ replacing $\Theta$.) We shall also say more briefly »Poincaré polygon». $\Pi(a)$ has the property that for each $b \in \Delta, \varphi(b) \cap \Pi(a) \neq \varnothing$. Further, if $b \in$ int $\Pi(a)$, then $\varphi(b)$ has only the point $b$ in common with $\Pi(a)$. The frontier of $\Pi(a)$ consists of the closure of the union of certain non-euclidean straight line arcs $\sigma$ and arcs $\gamma(\subset C)$. By a side of $\Pi(a)$ is meant a maximal $\sigma$ or $\gamma$ contained in fr $\Pi(a)$. Further,

$$
\mathrm{U}_{\tau \in \Theta} \tau[\Pi(a)]=\Delta,
$$

and for $\tau(\in \Theta)$ not the identity

$$
\operatorname{int} \Pi(a) \cap \operatorname{int} \tau[\Pi(a)]=\varnothing .
$$

There is no reason to restrict our attention to Poincaré fundamental polygons. We may as well consider more general »fundamental domains». We shall say that $D(\subset \Delta)$ is a non-euclidean convex fundamental domain of $\Theta$ provided that (1) $D$ is closed relative to $\Delta$ and is non-euclidean convex, (2) $\varphi(D)=\varphi(\Delta)$, (3) the restriction of $\varphi$ to int $D$ is univalent (or equivalently, int $D \cap \tau(\operatorname{int} D)=\varnothing, \quad \tau(\neq$ identity) $\in \Theta)$. Such a $D$ will be termed a non-euclidean convex fundamental polygon of $\Theta$, or more simply, a fundamental polygon of $\Theta$ provided that not only are the previous conditions fulfilled but also: (4) each component of $\Delta \cap$ fr $D$ is piecewise non-euclidean rectilinear, and (5) for each $z \in \Delta$ there exists

1) $\varphi^{-1}(E)$ denotes the antecedent of a set $E$ with respect to the map $\varphi$ with domain $\Omega$ specified by $(2.3)$. 
a neighborhood having points in common with $\tau D$ for only a finite set of $\tau \in \Theta$. The condition (5) is equivalent to the condition: (6) the restriction of $\varphi$ to $D$ tends to the ideal boundary of $\varphi(\Delta)$ as $|z| \rightarrow 1$. It is clear that a Poincaré fundamental polygon is a fundamental polygon in the present sense. The term »side» will be employed for fundamental domains in the same way as for Poincaré fundamental polygons.

3. A lemma concerning fundamental polygons for $\Theta$ of the second kind. We shall want information concerning the behavior of a fundamental polygon $\Pi$ of a group $\Theta$ in the neighborhood of a frontier point on $C$ at which $\Theta$ is properly discontinuous. The facts are quite straightforward but nevertheless require attention.

Lemma 3.1: Let $\Theta$ be properly discontinuous at $\zeta \in C$. Then $\bar{\Pi} \cap \varphi(\zeta)$ is a nonempty finite set. Further, either it reduces to an inner point of an arc of $C$ lying in fr $\Pi$, or else it consists of more than one point and in this latter case for each point of $\bar{\Pi} \cap \varphi(\zeta)$ one of the following alternatives occurs: (1) it is an endpoint of an arc $\gamma(\subset C)$ and an arc $\beta$ of a circle orthogonal to $C, \beta \subset \bar{\Delta}$, such that $\gamma \cup \beta \subset$ fr $I I$; (2) it is an endpoint of two distinct $\beta$ the union of which lies in fr $I I$.

Proof: It will be convenient to treat the equivalent problem where $\Theta$ is replaced by a group of Möbius transformations mapping $\{\operatorname{Im} z>0\}$ onto itself and properly discontinuous at each point of $\{\operatorname{Im} z>0\}$. We shall designate the group in question also by $\Theta$. (Of course, the definition of a fundamental polygon persists.) We take a point $c$ of the real axis where $\Theta$ is properly discontinuous and show first that there exists a neighborhood of $c$ which has points in common with $\tau(\Pi)$ for only a finite set of $\tau \in \Theta$. To that end, we introduce $\delta(>0)$ such that $\varphi(R)$ is a proper subset of $\varphi[\{\operatorname{Im} z>0\})$, where

$$
R=\{z|| \operatorname{Re} z-c \mid<7 \delta, 0<\operatorname{Im} z \leq 4 \delta\} .
$$

Every hyperbolic straight line segment, one endpoint of which is at a euclidean distance less than $\delta$ from $c$ and the other is at a euclidean distance less than $\delta$ from $c+6 \delta$, has the property that it lies on a euclidean circle whose center lies in the interval $(c+2 \delta, c+4 \delta)$ of the real axis and radius lies strictly between $2 \delta$ and $4 \delta$. A corresponding statement holds when one of the endpoints is at a euclidean distance less than $\delta$ from $c$ and the other is at a euclidean distance less than $\delta$ from $c-6 \delta$.

We assert that $\tau(\Pi)$ has points in common with $U(c, \delta)=\{|z-c|<\delta\}$ for only a finite set of $\tau \in \Theta$. In fact, by the choice of $\delta$ each $\tau(\Pi)$ must have points in $\{\operatorname{Im} z>0\}-R$. Hence if $\tau(\Pi) \cap U(c, \delta) \neq \varnothing$, then 
$E \cap \operatorname{int} \tau(\Pi) \neq \varnothing$, where $E$ is the intersection of $\{\operatorname{Im} z>0\}$ and the frontier of

$$
R_{1}=\{z|| \operatorname{Re} z-c \mid \leq 6 \delta, 0 \leq \operatorname{Im} z \leq 4 \delta\} .
$$

Since int $\tau(\Pi)$ is convex in the hyperbolic sense, it follows that there is a hyperbolic straight line segment $\lambda_{\tau} \subset$ int $\tau(\Pi)$ the endpoints of which are in $U(c, \delta)$ and $E$. If there were infinitely many distinct $\tau$ with the stated property, the endpoints of the $\lambda_{\tau}$ on $E$ would cluster either at $c+6 \delta$ or at $c-6 \delta$. Otherwise some point of $\{\operatorname{Im} z>0\}$ would have the property that each of its neighborhoods would contain points of $\tau(\Pi)$ for infinitely many distinct $\tau$. This is not possible. However, the endpoints of the $\lambda_{\tau}$ on $E$ cannot cluster at $c+6 \delta$. Otherwise for infinitely many distinct $\tau$ the endpoints in question would lie in $U(c+6 \delta, \delta)$, and consequently, for infinitely many distinct $\tau$ the point of maximum ordinate on the circle containing $\lambda_{\tau}$ would belong to $\lambda_{r}$ and also to the square

$$
R_{2}=\{z \mid 2 \delta<\operatorname{Re} z-c<4 \delta, 2 \delta<\operatorname{Im} z<4 \delta\} .
$$

But then we should be forced to conclude the existence of a point of $\{\operatorname{Im} z>0\}$ each neighborhood of which contains points of $\tau(\Pi)$ for infinitely many distinct $\tau$. This is not possible. Similarly the endpoints of the $\lambda_{\tau}$ on $E$ are seen not to cluster at $c-6 \delta$. Our assertion follows.

We next choose $\delta_{1}, 0<\delta_{1} \leq \delta$, so that $c$ belongs to the closure of each $\tau(\Pi)$ having points in common with $U\left(c, \delta_{1}\right)$. To continue, we note that each int $\tau(\Pi)$ is a Jordan region (in the sense of the topology of the extended plane). Let us consider a subarc $\alpha$ of the frontier of a $\tau(\Pi)$, where $\alpha \subset U\left(c, \delta_{1}\right)$ and $c$ is an endpoint of $\alpha$. We assert that some subarc of $\alpha$ having $c$ as an endpoint either is a segment of the real axis or else is a Jordan arc lying wholly in $\{\operatorname{Im} z>0\}$ save for the point $c$. Otherwise there would exist a sequence of subarcs of $\alpha$ lying in $\{\operatorname{Im} z>0\}$ save for endpoints that lie on the real axis and tend to $c$. But then the closure of the bounded Jordan region, the frontier of which consists of such a subarc and the segment of the real axis joining its endpoints, contains some $\sigma(\Pi), \sigma \in \Theta$. Hence there would exist arbitrarily small $\sigma(\Pi)$ in $U(c, \delta)$. This is not possible.

If some subarc $\alpha_{1}$ of $\alpha$ with endpoint $c$ lies wholly in $\{\operatorname{Im} z>0\}$ save for $c$, we may suppose that $\alpha_{1}$ less $c$ is a hyperbolic half line. In fact, under this condition for each $\tau$ for which $c \in \operatorname{fr} \tau(\Pi)$ we introduce one arc of $\tau(\Pi)$ with endpoint $c$ lying wholly in $\{\operatorname{Im} z>0\} \cap U(c, \delta)$ save for $c$ if $\tau(\Pi)$ contains a segment of the positive real axis having positive length with endpoint $c$, and otherwise we introduce two arcs of fr $\tau(\Pi)$ having only $c$ in common and lying wholly in $\{\operatorname{Im} z>0\} \cap U(c, \delta)$ 
save for $c$. We choose $\delta_{2}, 0<\delta_{2}<\delta$, so that any point of fr $\tau(\Pi)$ in $\{\operatorname{Im} z>0\} \cap U\left(c, \delta_{2}\right)$ lies on one of the chosen arcs associated with $\tau, \tau$ such that $c \in \tau(\Pi)$. We assert that there are no corners of a chosen arc in $U\left(c, \delta_{2}\right)$. If there were such a corner, say $q$, then the hyperbolic convexity of $\Pi$ would imply that $q$ would belong to fr $\tau(\Pi)$ for at least three distinct $\tau$. (We recall that the angular opening at a corner is less than $\pi$.) It follows that there would exist subarcs of two chosen arcs with endpoints $c$ and $q$ having in common in some neighborhood of $q$ no points distinct from $q$. These subarcs cannot meet at a point of $\{\operatorname{Im} z>0\}$ distinct from $q$; otherwise they would have subarcs having just their endpoints in common - the endpoints being $q$ and another point of $\{\operatorname{Im} z>0\}-$ and consequently, $\bar{\Pi}$ would be a bounded subset of $\{\operatorname{Im} z>0\}$ and hence $\Theta$ would not be of the second kind. It follows that the subarcs of the first clause of the preceding sentence form a closed Jordan curve $\gamma$ in $U(c, \delta)$ and that int $\tau(\Pi)$ would lie wholly in the bounded component of the complement of $\gamma$ and hence in $R$ for some $\tau$. The contradiction is apparent. The supposition of the first sentence of the present paragraph is valid.

The Lemma now follows readily.

4. Our next concern will be to reformulate the condition of Siegel in terms of $\varphi(\Delta)$ and the ramification function $\partial$.

We consider an arbitrary Riemann surface $F$ and a function $d$ on $F$ which takes positive integer values and is such that $\{d(q)>1\}$ clusters at no point of $F$. We say that $(F, d)$ satisfies the condition A provided that either (1) there does not exist a $C^{\prime \prime}$ conformal metric $\lambda$ of constant Gaussian curvature -4 on $F-\{d(q)>1\}$ subject to the condition that in terms of a local uniformizer $\sigma, \sigma(0)=q$,

$$
\lambda_{\sigma}(t)|t|^{1-[d(q)]^{-1}}
$$

possesses a finite positive limit at $0, \lambda_{\sigma}(t)|d t|$ being the local representation of the metric in terms of $\sigma, q \in F$; or else (2) such $\lambda$ exist and the supremum of the set of $\lambda$-areas of $F-\{d(q)>1\}$ is finite. Actually, if such a metric $\lambda$ exists, there is a maximal such $\lambda$. This fact will become clear subsequently (Th. 4.3). The $\lambda$ in question are real analytic, but use will not be made of this property.

We turn to the reformulation of Siegel's condition. Given a group $\Theta$, we let $F$ be $\varphi(\Delta)$ and $d$ the restriction of $\partial$ to $\varphi(\Delta)$. We have

Theorem 4.1: There exists a maximal $\lambda$ relative to $(F, d)$, say $\mu$. The hyperbolic area of each fundamental polygon is the $\mu$-area of $F-\{d(q)>1\}$. If the condition of Siegel is fulfilled for some fundamental 
polygon, then $(F, d)$ satisfies the condition $A$. If $(F, d)$ satisfies the condition $A$, each fundamental polygon has the same finite hyperbolic area and its frontier has a finite number of sides, none on $\{|z|=1\}$.

It is to be remarked that as a consequence of Siegel's hypothesis the frontier of every fundamental polygon has a finite number of sides. Of course, it is easy to see directly that every fundamental polygon has the same hyperbolic area (finite or infinite) for any given $\Theta$.

To establish Theorem 4.1 we note that the hyperbolic metric $\left(1-|z|^{2}\right)^{-1}|d z|$ restricted to $\Delta-\{n(z ; \varphi)>1\}$ defines via $\varphi$ a metric $\mu$ on $F-\{d(q)>1\}$ fulfilling the conditions imposed on a metric $\lambda$. Suppose that a competing $\lambda$ meeting the imposed conditions is considered. Let $\Lambda(z)|d z|$ and $M(z)|d z|$ denote the representations of $\lambda$ and $\mu$ given by the restriction of $\varphi$ to $\Delta-\{n(z ; \varphi)>1\}$. We have $M(z)=\left(1-|z|^{2}\right)^{-1}$. Further $\Lambda(z)|d z|$ is a $C^{\prime \prime}$ metric of constant curvature -4 and $A$ admits a continuous extension to $\Delta$. It follows that $\Lambda \leq M$ by a slightly generalized form of Ahlfors's extension [1] of the Schwarz lemma (cf. [6] p. 85). Hence $\lambda \leq \mu$.

Only the last assertion of the theorem calls for attention. It will be established once the following result is proved.

Theorem 4.2: If a fundamental polygon has a finite hyperbolic area, then its frontier has a finite number of sides none of which is on $\{|z|=1\}$. (Siegel's theorem for fundamental polygons.)

The proof is a very slight variant of Siegel's [10]. We first consider an arbitrary non-euclidean convex set $E \subset \Delta$, where $E$ is closed relative to $\Delta$, int $E \neq \varnothing$, and $E$ has finite hyperbolic area. We assert that $\Delta \cap \mathrm{fr} E$ has only a finite number of components. Otherwise we may assume that $0 \in$ int $E$ and that there are infinitely many hyperbolic triangles lying in $E$ each of which has 0 as a vertex and the remaining vertices on $\{|z|=1\}$ and which further have no interior points in common. These triangles may be taken as the hyperbolic triangles whose vertices are zero and the endpoints of a component of $\Delta \cap \mathrm{fr} E$ which subtends an angle less than (say) $\pi / 2$ at 0 . On using the formula for the area of a hyperbolic triangle in terms of the vertex angles we see that the area of $E$ would have to be infinite and we obtain a contradiction. Further fr $E$ does not contain an arc of $\{|z|=1\}$. Hence fr $E$ consists of the union of the above components and a finite set of points on $\{|z|=1\}$.

We now consider a fundamental polygon $\Pi$ having finite hyperbolic area. Again we may suppose that $0 \in$ int $\Pi$. We first note that for a given component of $\Delta \cap \mathrm{fr} \Pi$ and $\varepsilon>0$, at only a finite number of the vertices on $\Delta \cap \mathrm{fr} \Pi$ is the angle of the non-euclidean sector bounded by the sides issuing from the vertex and containing $\Pi$ less than $\pi-\varepsilon$. It suffices following Siegel to inscribe the hyperbolic triangles with vertices 0 and 
successive endpoints of a side of the component of $\Delta \cap \mathrm{fr} \Pi$ in question. Thereupon it is easy to control the vertex angles with the aid of the formula for the area of a hyperbolic triangle. Suppose that there are infinitely many vertices on a component. Let $\left(z_{k}\right)_{1}^{\infty}$ be a sequence of successive vertices proceeding in one sense along the component. For the hyperbolic triangle with vertices $0, z_{k}, z_{k+1}$ we denote the angle at $z_{k}$ by $\alpha_{k}$, at $z_{k+1}$ by $\beta_{k}$ and the angle at 0 by $\gamma_{k}$. For each positive integer $n$, we have

$$
\sum_{k=1}^{n}\left(\pi-\alpha_{k+1}-\beta_{k}\right)+\left(\pi-\alpha_{1}-\beta_{n+1}\right) \leq \mathrm{A}+\sum_{k=1}^{n+1} \gamma_{k},
$$

where $A$ is the hyperbolic area of $\Pi$. Hence

$$
\sum_{k=1}^{n}\left(\pi-\alpha_{k+1}-\beta_{k}\right) \leq A+2 \pi+\beta_{n+1}<A+3 \pi .
$$

We conclude that $\lim _{k \rightarrow>\infty}\left(\alpha_{k+1}+\beta_{k}\right)=\pi$. Our assertion follows, the case where there are only a finite number of vertices on a component of $\Delta \cap \mathrm{fr} I I$ being obvious.

The proof is now completed by noting first that since there are only a finite number of components of $\Delta \cap \mathrm{fr} \Pi$, given $\varepsilon>0$ there are only a finite number of vertices at which the angle is less than $\pi-\varepsilon$. If infinitely many vertices were present, there would exist a vertex with associated angle $\omega, 3 \pi / 4<\omega<\pi$, such that each equivalent point of fr $\Pi$ is either not a vertex or else is a vertex whose associated angle lies strictly between $3 \pi / 4$ and $\pi$. On summing the angles associated with the points of fr $\Pi$ equivalent to the vertex in question, we should obtain a sum of the form $2 \pi / n$ where $n$ is a positive integer, the angle $\pi$ being associated with a point, not a vertex, equivalent to the given vertex. If the number of points of $\mathrm{fr} \Pi$ equivalent to the given vertex is $m$, then $3 \pi m / 4<2 \pi / n<\pi m$ and hence $2<m n<8 / 3$. The contradiction is manifest.

It will be observed by the reader conversant with the proof of [10] that we have introduced a slight modification of the Siegel argument. Here we show the finiteness of the number of components and then the finiteness of the number of vertices on the components. The reverse procedure is followed in [10]. There however one is led to treat the limiting behavior of such angles as $\beta_{n}$ for $n \rightarrow+\infty$. Such considerations are eliminated by the present reversal of the argument.

A treatment of Siegel's theorem as originally stated for Poincaré polygons is to be found in [11].

We now turn to the situation of an unrestricted $(F, d)$ and characterize the $(F, d)$ satisfying the condition $A$ with the aid of Siegel's theorem. 
The following results are well known from the classical theories of Riemann surfaces and automorphic groups:

Given $(F, d)$. We suppose that $F$ is not conformally equivalent to the extended plane or $\{d(q)>1\}$ contains at least three points. There exists a conformal map $\psi$ of one of the standard simply connected regions - the extended plane, the finite plane, $\Delta$-onto $F$ fulfilling the following conditions: (1) The restriction of $\psi$ to

$$
\psi^{-1}[F-\{d(q)>1\}]
$$

is a covering of the region $F-\{d(q)>1\}$. (2) If $d(q)>1$, there exists a region $\omega, q \in \omega$, such that the restriction of $\psi$ to a component of $\psi^{-1}(\omega)$ has constant valence $d(q)$ on $\omega$.

If $\psi_{1}$ and $\psi_{2}$ both satisfy the stated conditions for a given $(F, d)$, then they both have the same domain and there exists a conformal automorphism, $\alpha$, of the domain such that $\psi_{2}=\psi_{1} \circ \alpha$.

Each $\psi$ is automorphic with respect to a group of conformal automorphisms of its domain such that for each $p \in F, \psi^{-1}(\{p\})$ is an orbit of the group.

If the domain of $\psi$ is the extended plane, $F$ is conformally equivalent to the extended plane and $\{d(q)>1\}$ contains precisely three members. If the domain of $\psi$ is the finite plane, then $F$ is conformally equivalent to one of the following: extended plane, finite plane, punctured plane, a torus, and $\{d(q)>1\}$ contains at most four points.

With the aid of these results and Siegel's theorem we are in a position to establish

Theorem 4.3: A pair $(F, d)$ satisfies the condition $A$ if and only if $F$ is conformally equivalent to a compact Riemann surface less a finite set of points and $\{d(q)>1\}$ is a finite set.

Proof: Suppose that $(F, d)$ satisfies the condition $A$. We put aside the trivial case where $F$ is conformally equivalent to the extended plane and $\{d(q)>1\}$ has at most two elements. We may further suppose that the domain of the associated $\psi$ is $\Delta$. In this case we see that the condition of Siegel is fulfilled by the fundamental polygons of the group of conformal automorphisms $\Gamma$ of $\Delta$ leaving $\psi$ invariant. Since $\psi=\theta \circ \varphi$, where $\theta$ is a univalent conformal map of $\varphi(\Delta)$ onto $F(\varphi$ referring to $\Gamma)$, the theorem of Siegel leads to the stated conclusion inasmuch as the finiteness condition on the number of sides of a fundamental polygon and the fact that none is on $C$ imply that $\varphi(\Delta)$ is conformally equivalent to a compact Riemann surface less a finite set of points and $\{\partial(q)>1\}$ is finite. It is to be emphasized in this connection that the set of points on the frontier of a fundamental polygon having a finite number of sides at which the multiplicity of $\varphi$ is two is finite. 
We now proceed in the opposite direction. Here, too, it is sufficient to consider the case where $F$ is not conformally equivalent to the extended plane or $\{d(q)>1\}$ contains at least three points. Further, we may restrict our attention to the case where the domain of $\psi$ is $\Delta$. For in all cases put aside there does not exist a metric $\lambda$ satisfying the stated conditions. In the case to which we are restricting attention, the metric induced as above from the hyperbolic metric on $\Delta$ by $\psi$ yields $\mu$, the maximal $\lambda$ subject to the stated conditions. If $\theta$ is a univalent conformal map of $\Delta-\{0\}$ into $F$ which tends to a boundary element of $F$ as $z \rightarrow 0$, then $\mu_{\theta}(z)$ satisfies

$$
\mu_{\theta}(z) \leq \frac{1}{|z| \log \frac{1}{|z|}}+O(1)
$$

$\mu_{\theta}(z)|d z|$ being the representation of $\mu$ corresponding to the uniformizer $\theta$. It follows that the $\mu$-area of $F-\{d(q)>1\}$ is finite.

5. Let us now consider a group $\Theta$ of the second kind and let us now take $F=\varphi(\Omega)$ and $d=\partial$. We shall establish the following theorem.

Theorem 5.1: Let $\Pi^{*}$ denote the union of a fundamental polygon $I I$, its image with respect to the inversion $z \rightarrow 1 / \bar{z}$, and $\operatorname{fr} \Pi \cap C \cap \Omega$. If the pair $(\varphi(\Omega), \partial)$ satisfies the condition $A$, then either $C-\Omega$ contains at most two points or otherwise the area of each $\Pi^{*}$ in the sense of the maximal conformal metric of constant curvature -4 on $\Omega$ is finite. If $C-\Omega$ contains at most two points or the area condition holds for some $\Pi^{*}$, then $(\varphi(\Omega), \partial)$ satisfies the condition $A$.

The proof of this theorem may be based on the following observations. First, if $C-\Omega$ contains at most two points, there does not exist a metric $\lambda$. Second, if $C-\Omega$ contains more than two points, then ('), the maximal conformal metric of constant curvature -4 on $\Omega$, induces via $\varphi$ the maximal $\lambda$ on $q(\Omega)-\{\partial(q)>1\}$ satisfring the stated conditions and the area (finite or infinite) of $q(\Omega)-\{\partial(q)>1\}$ with respect to the maximal $\lambda$ is equal to the $\mu$-area of each $\Pi^{*}$. The second observation yields the first assertion of the theorem. Together the two observations lead to the second assertion. The details are readily supplied.

6. We now turn to the counterpart of the Siegel theorem for $\Theta$ of the second kind formulated in terms of condition $A$.

Theorem 6.1: If $\Theta$ is a group of the second kind such that $(\varphi(\Omega), \partial)$ ) satisfies condition $A$, then each associated fundamental polygon has a finite number of sides. 
We put aside the trivial case where $\Theta$ reduces to the identity and as a first step in the proof we consider the behavior of $\varphi$ on the components of $\Omega \cap C$. It is easy to see that either $\varphi$ is univalent on such a component $\alpha$ or else $\alpha$ is mapped onto itself by some member of $\Theta$ distinct from the identity and that in the latter case $\varphi(\alpha)$ is a regular analytic closed Jordan curve. In the former case $\varphi(z)$ tends to the ideal boundary of $\varphi(\Omega)$ as $z$ tends along $\alpha$ to either of its endpoints. In fact, if this were not so, there would exist a sequence $\left(z_{k}\right)_{1}^{\infty}$ of points of $\alpha$ tending to an endpoint of $\alpha$ and such that $\left(\varphi\left(z_{k}\right)\right)_{1}^{\infty}$ tended to a point $q \in \varphi(\Omega \cap C)$. But then $q=\varphi(w)$, $w \in \beta$, where $\beta$ is a component are of $\Omega \cap C$. Let $r>0$ be so small that $\varphi$ is univalent on $\overline{U(w, r)} \subset \Omega$. For $k$ sufficiently large $\varphi\left(z_{k}\right) \in \varphi[U(w, r)]$. We fix such a $k$, say $l$, and let $\tau \in \Theta$ be such that $\tau\left(z_{l}\right) \in U(w, r)$. We have $\left.\tau^{-1} \overline{[U(w, r)} \cap \beta\right] \subset \alpha$. Hence $\left.\phi \overline{[U(w, r)} \cap \beta\right] \subset \varphi(\alpha)$. For $k$ sufficiently large $\left.z_{k} \notin \tau^{-1} \overline{[U(w, r)} \cap \beta\right]$ and at the same time $\varphi\left(z_{k}\right) \in \varphi[U(w, r) \cap \beta]$. Hence $\varphi$ would not be univalent on $\alpha$. Our hypothesis is contradicted. The assertion follows.

Thanks to Theorem 4.3 we may consider $\varphi(\Omega)$ as $G-\left\{a_{1}, \ldots, a_{n}\right\}$ where $G$ is a compact Riemann surface and the $a_{k}$ are distinct points of $G, n \geq 0$. We now show that no $a_{k}$ belongs to $\overline{\varphi(\Omega \cap C)}$. We may suppose $n \geq 1$, the case where $n=0$ being trivial. To that end, we consider a closed Jordan disk $D(\subset G)$ containing $a_{j}$ in its interior, $a_{k} \notin D, k \neq j$, and such that $\psi$ is not ramified over any point of int $D$.

Suppose that for some component $\lambda$ of $\Omega \cap C$ we have $\phi(\alpha) \subset \operatorname{int} D$. Then in this case $\overline{\varphi(x)}$ is a Jordan curve in int $D$. The component $\delta$ of int $D-\overline{\varphi(\alpha)}$ the closure of which lies in int $D$ does not contain points of $\varphi(\Omega \cap C)$, for otherwise the connectedness of $\varphi(\Delta)$ or of $\varphi(\{|z|>1\})$ would be violated. If $a_{j} \notin \delta$, then the restriction of $\varphi$ to a component of $\varphi^{-1}(\delta)$ would be univalent. The component in question would have to be either $\Delta$ or $\{|z|>1\}$ and $\Theta$ would reduce to the identity. This is impossible. Hence $a_{j} \in \delta$ and consequently $a_{j} \notin \overline{\varphi(\Omega \cap C)}$.

Our task is reduced to considering the case where for no $\alpha$ is it true that $\varphi(\alpha) \subset \operatorname{int} D$. Only a finite set of $\varphi(\alpha)$ have points in common with int $D$. Otherwise there would be an infinite set $E$ of points on fr $D$ each point of which belongs respectively to a distinct $\varphi(x)$. Let $\varphi(c) \in$ fr $D$ denote a cluster point. We obtain a contradiction when we consider $\varphi\left(x_{0}\right)$, where $\alpha_{0}$ is the component arc containing $c$. For $\varphi\left(\alpha_{0}\right)$ would contain infinitely many points of $E$. However distinct $\varphi(\alpha)$ are disjoint. If $a_{j}$ belonged to $\overline{\varphi(\Omega \cap C)}$, then $a_{j}$ would be an endpoint of some $\varphi(\alpha)$. Further, there would exist a closed Jordan disk $D_{1}(\subset$ int $D)$ such that $\left[D_{1} \cap \varphi(\Omega \cap C)\right] \cup\left\{a_{j}\right\}$ consisted of the union of a finite number of Jordan arcs having only $a_{j}$ in common, the endpoints of each arc being $a_{j}$ and 
a point of fr $D_{1}$ and the remaining points lying in int $D_{1}$. There would have to be at least two such arcs, otherwise some point of $\varphi(\Delta)$ and some point of $\varphi(\{|z|>1\})$ could be connected by an arc not intersecting $\varphi(\Omega \cap C)$. Consider a component, $K$, of int $D_{1}$ less these arcs. We suppose, as we may, that $K \subset \varphi(\Delta)$. It follows from considerations concerning the boundary behavior of conformal maps of Jordan regions and the Schwarz reflexion principle that fr $K_{1}$ contains a free arc $(\subset C)$ which contains a point $c \in C-\Omega$, where $K_{1}$ is a component of $\varphi^{-1}(K)$. It is to be noted that the restriction of $\varphi$ to $K_{1}$ is univalent. We conclude that $\varphi$ would have to be properly discontinuous at $c$. But this is not possible. Consequently, $a_{j} \notin \overline{\varphi(\Omega \cap C)}$. (We shall see that this result remains valid for arbitrary $\Theta$ of the second kind. The present discussion of the special case under consideration is given solely to prevent unduly burdening the exposition of the first part of this paper.)

It now follows that each $\varphi(\alpha)$ is a closed Jordan curve. Further the distinct $\varphi(\alpha)$ are finite in number. Hence, a fundamental polygon has only a finite number of sides on $C$, as may be seen by appeal to Lemma 3.1. Each lies in $\Omega$.

We recall that we have put aside the trivial case where $\Theta$ reduces to the identity. We note that thanks to the non-euclidean convexity of a fundamental polygon the hyperbolic straight line having the same endpoints as a side of $\Pi$ lying in $C$ is contained in $\Pi$. This hyperbolic line lies in int $\Pi$, or else int $\Pi$ is the bounded region the frontier of which consists of the above side of $C$ and the hyperbolic straight line. Nothing remains to be shown in the latter case. There are just two sides.

In the former case we proceed as follows. Every hyperbolic line so associated with a side of $\Pi$ on $C$ lies in int $\Pi$. We obtain a set $\Pi_{1}$ by removing from $\Pi$ each of the bounded regions the frontiers of which consist of a side of $I I$ on $C$ and the hyperbolic straight line joining the endpoints of the side. Each such region is contained in int $\Pi$. The set $\Pi_{1}$ is a non-empty non-euclidean convex polygon. The term "side" applied to $\Pi_{1}$ is to have the same meaning relative to $\Pi_{1}$ as that given before for a side of $\Pi$. The term "hyperbolic area" is now to be taken in the sense of the hyperbolic metric in $\Delta$. For each point $c \in \overline{\Pi_{1}} \cap \Omega \cap C$ we introduce a non-euclidean triangle $T(c) \subset \Pi_{1}$, of which the vertices are $c$ and inner points of the two sides of $\Pi_{1}$ which have $c$ as an endpoint. We let $\Pi_{2}$ denote $\Pi_{1}$ less the union of the $T(c)$. It is easy to see that

$$
\overline{\varphi\left(\Pi_{2}\right)} \cap \varphi(\Omega \cap C)=\varnothing .
$$

Let $\mu$ denote the conformal metric of constant curvature -4 induced on $\varphi(\Delta)-(\partial(q)>1\}$ from the hyperbolic metric on $\Delta$ by $\varphi$. From 
(6.1) we conclude that the $\mu$-area of $\varphi\left(\operatorname{int} \Pi_{2}\right)$ is finite. Consequently, the hyperbolic area of $\Pi_{2}$ is finite and hence so is the hyperbolic area of $\Pi_{1}$.

The argument of Siegel [9] may now be transcribed. The present proof parallels that of Theorem 4.2. We first show that $\Delta \cap \mathrm{fr} \Pi_{1}$ has only a finite number of components. We restrict our attention, as we may, to the case where there is more than one. We choose $b \in \operatorname{int} \Pi_{1}$ and consider the non-euclidean triangles the vertices of which consist of $b$ and the endpoints of a component of $\Delta \cap \mathrm{fr} \Pi_{1}$. Distinct such triangles corresponding to components intercepting a non-euclidean angle less than $\pi$ at $b$ do not have any interior points in common. Further the union of all the so restricted triangles lies in $\Pi_{1}$. On using the fact that the area of a non-euclidean triangle is $\pi$ less the sum of the vertex angles, we conclude that $\Delta \cap \operatorname{fr} \Pi_{1}$ has only a finite number of components since the sum of the areas of the triangles is finite and the sum of the vertex angles at $b$ does not exceed $2 \pi$. It is now easily seen that $C \cap$ fr $\Pi_{1}$ has only a finite number of points; otherwise the area would be infinite.

If a component of $\Delta \cap$ fr $\Pi_{1}$ contains infinitely many sides, then as is readily seen by the reasoning of Siegel the angles formed by successive sides tend to $\pi$ as their vertices tend to an endpoint of the component. It suffices to consider the non-euclidean triangles with vertices $b$ and the end points of a side and repeat the area argument. Hence if fr $\Pi_{1}$ had infinitely many sides, the vertex angles would exceed $3 \pi / 4$ for all but a finite number of vertices. We are led to the same contradiction as in Theorem 4.2. Hence fr $\Pi_{1}$ has a finite number of sides and, a fortiori, so does fr $\Pi$.

7. Thanks to Theorem 4.2, Theorem 5.1 and Theorem 6.1 , it is possible to give the proof which was referred in $\S 1$ to the present section. We obtain a second proof, of course, via Theorem 1.1 to be established in $\S 10$.

We note that if $\Theta$ is of the first kind, then a fundamental polygon has no sides on $C$ and it is classical that if such a polygon has only a finite number of sides, its hyperbolic area is finite. The theorem of Siegel as given by Theorem 4.2 applies. If $\Theta$ is of the second kind and $\Pi$ is a fundamental polygon with a finite number of sides, we proceed as follows. If $C-\Omega$ has at most two members, Theorem 5.1 is applicable and shows that condition $A$ holds. On examining $\Pi^{*}$ in the remaining case we see that its area in the sense of the maximal $C^{\prime \prime}$ conformal metric of constant curvature -4 on $\Omega$ is finite. It suffices to note that the sides of $\Pi$ on $C$ also lie in $\Omega$ and that the area of a hyperbolic triangle in $\Delta$ in the sense of this metric, being dominated by the area in the hyperbolic sense, is finite so that contributions from neighborhoods of cusps are harmless. The condition $A$ is fulfilled and Theorem 6.1 applies. 
8. The pairs $\left(\varphi(\Delta), \partial_{1}\right)$, where $\partial_{1}$ is the restriction to $\varphi(\Delta)$ of $\partial$, for $\Theta$ with $(\varphi(\Delta), \partial)$ or $(\varphi(\Omega), \partial)$ satisfying condition $A$ may be characterized conformally as the following theorem shows. Its proof, which depends in part on the classical results concerning branched coverings of the extended plane, is readily supplied at this point and will be omitted.

Theorem 8.1: Let there be given a pair $(F, d)$. A necessary and sufficient condition that there exist a $\Theta$ of the first kind such that (1) there exists a univalent conformal map $\psi$ of $\varphi(\Delta)$ onto $F$ satisfying $d \circ \psi=\partial,(2)$ $(\varphi(\Delta), \partial)$ satisfies condition $A$ is that $F$ be conformally equivalent to a compact Riemann surface $G$ less a finite set of points, that $\{d(q)>1\}$ be finite, and that

$$
\sum_{q \in F}\left\{1-[d(q)]^{-1}\right\}>-(\chi+m)
$$

where $\chi$ is the Euler characteristic of $G(\chi=2 g-2, g=$ genus of $G)$ and $m$ is the number of boundary elements of $F$. A necessary and sufficient condition that there exist a $\Theta$ of the second kind such that (1) there exists a conformal map $\psi$ of $\varphi(\Delta)$ onto $F$ satisfying $d \circ \psi=\partial_{1},(2)(\varphi(\Omega), \partial)$ satisfies condition $A$ is that $F$ be a hyperbolic Riemann surface having finite topological characteristics and that $\{d(q)>1\}$ be finite.

We remark: if $\left(\varphi(\Delta), \partial_{1}\right)$ satisfies condition $A$, then $\Theta$ is of the first kind. This observation follows from Theorem 4.3 and the fact that $\varphi(\Delta)$ is hyperbolic for $\Theta$ of the second kind.

9. It is possible to replace the sufficient condition of Theorem $4.3 \mathrm{by}$ a less stringent one in the case where $F=\varphi(\Omega)$ and $d=\partial, \Theta$ being given. Here $\Omega$ is the component containing $\Delta$ of the set of points at which $\Theta$ is properly discontinuous. We have

Theorem 9.1: If $\varphi(\Omega)$ has finite topological characteristics (genus and number of Kerékjártó boundary elements) and $\{\partial(q)>1\}$ is finite, then $(\varphi(\Omega), \partial)$ satisfies condition $A$.

Suppose that $\Theta$ is of the first kind. It suffices to show that $\varphi(\Delta)$ is parabolic for then each of its boundary components is necessarily isolated, planar and pointlike. If $\varphi(\Delta)$ were hyperbolic, there would exist a univalent conformal map $\psi$ of $\varphi(\Delta)$ onto a region of a compact Riemann surface the frontier of which consists of a finite number of components, where each either is a regular analytic Jordan curve or reduces to a point and at least one does not reduce to a point. Since $\psi \circ \varphi$ restricted to $\varphi^{-1}\{\partial(q)=1\}$ is a covering of $\psi\{\partial(q)=1\}$, it follows on considering a component of the antecedent of a simply-connected Jordan region $\omega \subset \psi \circ \varphi(\Delta)$, where fr $\omega$ contains an arc lying in fr $\psi \circ \varphi(\Delta)$, that for some $\eta,|\eta|=1$, and $r>0, \psi \circ \varphi$ and hence $\varphi$ would be univalent in $U(\eta, r) \cap \Delta$. 
This would force $\Theta$ to be of the second kind. The contradiction is manifest.

Since $\varphi(\Delta)$ is parabolic and hence the boundary components are isolated, planar and pointlike, the associated maximal $C^{\prime \prime}$ conformal metric $\mu$ of constant curvature -4 (such a metric always exists for $\Theta$ of the first kind) is such that the $\mu$-area of $\varphi(\Delta)-\{\partial(q)>1\}$ is finite.

Suppose now that $\Theta$ is of the second kind. We restrict our attention to the case where $C-\Omega$ contains more than two points and consequently is perfect - the situation where $C-\Omega$ contains at most two points is immediate. We now let $\psi$ denote a conformal universal covering of $\Omega$ with domain $\Delta$. Since $\Gamma$, the group of conformal automorphisms leaving $\psi$ invariant, is such that $\Theta(T)$ is of the first kind, $C-\Omega$ being totally disconnected, $\Theta\left(\Gamma_{1}\right)$ is also of the first kind, where $\Gamma_{1}$ is the group of conformal automorphisms of $\Delta$ leaving $\varphi \circ \psi$ invariant. Let $\varphi_{1}$ denote the orbit map associated with $\Gamma_{1}$ and let $F_{1}=\varphi_{1}(\Delta)$. We have

$$
\varphi \circ \psi=\theta \circ \varphi_{1},
$$

where $\theta$ is a univalent conformal map of $F_{1}$ onto $\varphi(\Omega)$. It is to be noted here that if $\varphi\left[\psi\left(z_{1}\right)\right]=\varphi\left[\psi\left(z_{2}\right)\right]$, then $\psi\left(z_{2}\right)=\sigma\left[\psi\left(z_{1}\right)\right], \sigma \in \Theta$, and also $\sigma \circ \psi=\psi \circ \tau, \tau \in \Gamma_{1}$, so that $z_{2}=\tau_{1}\left(z_{1}\right), \tau_{1} \in \Gamma_{1}$. It follows from the first part of the proof that $F_{1}$ is conformally equivalent to a compact Riemann surface less a finite set of points. The theorem follows.

10. Finitely generated $\Theta$. We show

Theorem 10.1: A group $\Theta$ is finitely generated if and only if the associated $(\varphi(\Omega), \partial)$ satisfies condition $A$. Here $\Omega$ is the component containing $\Delta$ of the set of points at which $\Theta$ is properly discontinuous. ${ }^{1}$

Before turning to the proof we remark that Theorem 10.1 taken with the theorem of Siegel for fundamental polygons and Theorem 6.1 yields Theorem 1.1. It is to be observed that if there exists a fundamental polygon with a finite number of sides, then condition $A$ is fulfilled.

The "if» part of Theorem 10.1 is readily disposed of. Suppose that condition $A$ is fulfilled by $(\varphi(\Omega), \partial)$. We fix $a \in \Delta, n(a ; \varphi)=1$, and introduce a finite generating set of path classes $K_{1}, \ldots, K_{m}$ for the fundamental group, $\Pi_{1}$, of $\varphi(\Delta)-\{\partial(q)>1\}$ based at $\varphi(a)$. A homomorphism of $\Pi_{1}$ onto $T$ is defined by assigning to each $K \in \Pi_{1}$ the element $\tau_{k} \in \Gamma$ such that for each path $\in K$ the lifting with respect to $\phi$ having initial point $a$ has terminal point $\tau_{k}(a)$. Hence $\Gamma$ is finitely generated.

1 Added January 24, 1964: I have been informed by Professor Ahlfors that this theorem is essentially known but that hitherto no proof has been published. He has obtained the corresponding result for Klein gronps. 
To establish the »only if» part, we first show that $\varphi(\Delta)$ has finite topological characteristics and that $\left\{\partial_{1}(q)>1\right\}$ is finite, where $\partial_{1}$ is the restriction of $\partial$ to $\varphi(\Delta)$. In the case of $\Theta$ of the second kind it follows that $\varphi(\Omega)$ has finite topological characteristics and that $\{\partial(q)>1\}$ is finite. The proof of the theorem under consideration is completed by appeal to Theorem 9.1.

The verification of the finiteness properties of $\varphi(\Delta)$ and $\partial_{1}$ will be based on the following lemma, a proof of which may be given with the aid of the monodromy theorem. The details of the proof will be omitted.

Lemma 10.1: Let $f$ denote a conformal map of $\Delta$ into a Riemann surface $H$ and let $g$ denote a conformal map of a Riemann surface $G$ onto $H$. Suppose that the following conditions are fulfilled: (1) There exists a discrete set $E \subset H$ such that the restriction of $g$ to $g^{-1}(H-E)$ is a covering of $H-E$. (2) For each $a \in E$ there exists a positive integer $v(a)>1$ such that for every sufficiently small open disk $\delta$ containing a the restriction of $g$ to a component of $g^{-1}(\delta)$ has valence $v(a)$ on $\delta$. (3) Whenever $f(z) \in E$, $n(z ; f)$ is a multiple of $v[f(z)]$. Then there exists a conformal map of $\Delta$ into $G$, say $\psi$, such that $f=g \circ \psi$.

For the remainder of the present section we shall understand by $\varphi$ the $\operatorname{map} z \rightarrow \varphi(z), z \in \Delta$.

We put aside the trivial case where $\Theta$ reduces to the identity. Let $\alpha_{1}, \ldots, \alpha_{n}$ denote a finite set of distinct elements of $\Theta$, each distinct from the identity, which generate $\Theta$ considered as a semigroup. We assume, as we may, that 0 is not a fixed point of any $\tau(\in \Theta)$ distinct from the identity. Let

$$
K=\bigcup_{i=1}^{n} \varphi\left(\left\{\alpha_{i}(0) t \mid 0 \leq t \leq 1\right\}\right)
$$

Suppose that $F=\varphi(4)$ has infinite genus. Let $R$ denote a relatively compact region of $F$ containing $K$ the frontier of which is also fr (ext $R$ ) and consists of a finite number of mutually disjoint closed Jordan curves. There is a component, $\mathfrak{C}$, of ext $R$ which is of infinite genus. We define a 2-sheeted covering, $g$, of $F$ by introducing a non-separating closed Jordan curve, $\gamma$, in $\mathfrak{C}$ and joining two copies of $F$ slit along $\gamma$ in the standard manner. Let $G$ denote the domain of $g$. Applying Lemma 10.1, we obtain $\varphi=g \circ \psi$, where $\psi$ is a conformal map of $\Delta$ onto $G$. That $G=\psi(\Delta)$ is a consequence of the fact that $\varphi$ and hence, $\psi$ do not have any asymptotic points (in $F$ or $G$ respectively). Consequently, it follows from the definition of $g$ that $\varphi^{-1}(R)$ has more than one component. Since $\alpha_{1}, \ldots, \alpha_{n}$ generate $\Theta$ as a semigroup, for each $\tau \in \Theta$ there exists a path in $\Delta$ joining 0 to $\tau(0)$ whose image with respect to $\varphi$ lies in $K$. 
Indeed, if $\tau=\alpha_{i(1)} \ldots \alpha_{i(m)}$ and $m>1$, it suffices to consider the path formed by joining the following arcs in succession:

$$
\begin{gathered}
\left.\overline{0 \alpha_{i(1)}(0)}, \quad \alpha_{i(1)} \overline{\left[0 \alpha_{i(2)}(0)\right.}\right], \alpha_{i(1)} \alpha_{i(2)}\left[\overline{\left.\left.0 \alpha_{i(3)}\right) 0\right)}\right], \ldots, \\
\alpha_{i(1)} \alpha_{i(2)} \ldots \alpha_{i(m-1)}\left[\overline{\left.0 \alpha_{i(m)}(0)\right]} .\right.
\end{gathered}
$$

The notation $" \overline{a b} »$ denotes a straight line segment as usual. We conclude that $\varphi^{-1}(R)$ is connected. The contradiction is manifest and we conclude that the genus of $\varphi(\Delta)$ is finite.

Each of the remaining parts of the verification may be carried out along similar lines. We consider the boundary components of $\varphi(\Delta)$ in the sense of Kerékjártó. Suppose that there are infinitely many. We take $R$ so that each component of ext $R$ is planar. At least one of these components, say $(5$, is neither simply-connected nor doubly-connected. We obtain a 2 -sheeted covering, $g$, of $F$ having the property that $g^{-1}(R)$ is not connected as follows. We suppose that $F$ is embedded in a compact Riemann surface, $F_{1}$, having the same genus as $F$, and define a 2 -sheeted conformal map, $g_{1}$, with image $F_{1}$ as follows. We introduce a Jordan arc, $\gamma_{1}$, where $\gamma_{1}$ lies in the component of $F_{1}-\bar{R}$ containing $C$ and joins points of distinct components of $F_{1}-F$. We thereupon join two copies of $F_{1}-\gamma_{1}$ along $\gamma_{1}$ in the standard manner and obtain a map of valence two with image $F_{1}$, which is ramified over the endpoints of $\gamma_{1}$. The desired covering, $g$, of $F$ is obtained as the restriction of $g_{1}$ to $g_{1}^{-1}(F)$. It is to be noted that $g_{1}^{-1}(F)$ is connected as a consequence of the choice of $g_{1}$. Further, as in the preceding paragraph, $g^{-1}(R)$ has two components. The remainder of the argument continues as above and we conclude that $F$ has a finite number of Karékjártó boundary elements.

Finiteness of $\left\{\partial_{1}(q)>1\right\}$. We assume, as we now may, that $F$ is a region of a compact Riemann surface $F_{1}$, where $F_{1}-F$ consists of the union of a finite family of mutually disjoint sets that reduce to points or are homeomorphs of closed disks. Suppose that $\left\{\partial_{1}(q)>1\right\}$ is infinite. We choose $R$ so that each component of $F_{1}-\bar{R}$ is the homeomorph of a disk which contains precisely one component of $F_{1}-F$. For some component, $\mathfrak{C}$, of $F-\bar{R}$, we have $\partial_{1}(q)>1$ at infinitely many points of $\mathfrak{C}$. Let $a(\in \mathbb{C})$ be such that $\partial_{1}(a)>1$, let $\mathfrak{S}_{1}$ denote the component of $F_{1}-\bar{R}$ containing $\mathfrak{C}$, and let $b \in \mathfrak{S}_{1}-\mathfrak{S}$. Let $\gamma$ denote a Jordan arc in $\mathfrak{r}_{1}$ with endpoints $a$ and $b$. We introduce $\partial_{1}(a)$ copies of $F_{1}-\gamma$, taken in cyclic order, and join them along $\gamma$, welding a preferred edge of each copy to the other edge of the successor copy. We obtain a conformal map, $g_{1}$, of constant valence $\partial_{1}(a)$, having image $F_{1}$, ramified over $a$ and $b$ but nowhere else, the multiplicity of $g_{1}$ at the antecedents of $a$ 
and $b$ being $\partial_{1}(a)$. Restricting $g_{1}$ to $g_{1}^{-1}(F)$ we obtain the desired $g$. The remainder of the above argument may now be paraphrased. Our assertion follows.

11. In the situation of Siegel's theorem where $\Theta$ are considered for which a fundamental polygon $\Pi$ has finite hyperbolic area, $\Theta$ is of the first kind and the presence of a point of $\bar{\Pi}$ on $C$ is equivalent to the existence of a parabolic member of $\Theta$ (cf. [10], p. 45). In the case of $\Theta$ of the second kind for which the associated $(\varphi(\Omega), \partial)$ satisfy condition $A$ the facts are palpably different. Here the wellknown phenomenon of an adventitious cusp may occur at a point of $\Omega \cap C$. It suffices to consider $\Theta$ generated by the transformations $\sigma$ and $\tau$, where

$$
\begin{aligned}
& \frac{\sigma(z)-\varepsilon}{\sigma(z)+\varepsilon}=\lambda \frac{z-\varepsilon}{z+\varepsilon}, \\
& \frac{\tau(z)-\bar{\varepsilon}}{\tau(z)+\bar{\varepsilon}}=\lambda \frac{z-\bar{\varepsilon}}{z+\bar{\varepsilon}},
\end{aligned}
$$

$\varepsilon=e^{\pi i / 8}, \lambda=-(1+\varepsilon)^{2} /(1-\varepsilon)^{2}$. In this example, the Poincaré polygon $\Pi(0)$ has adventitious cusps at 1 and -1 . Nevertheless, there is a perfect correlation between conjugate classes of maximal cyclic subgroups generated by parabolic transformations of a completely arbitrary $\Theta$ and finite cycles of cusps of the associated fundamental polygons. As far as I am aware, this question has not been hitherto treated in full generality. In studying it we shall make use of the results of our paper [5], in which is developed the relation between conjugate classes of maximal cyclic subgroups of parabolic transformations and the isolated pointlike planar boundary elements of $\varphi(\Delta)$ having deleted neighborhoods free of $\{\partial(q)>1\}$. However, before we turn to this question, it will be convenient to investigate the isolated planar boundary elements of $\varphi(\Omega)$ having a deleted neighborhood free of $\{\partial(q)>1\}, \Omega$ denoting as above the component containing $\Delta$ of the set of points at which $\Theta$ is properly discontinuous. Such isolated planar boundary elements will be termed admitted. We first establish the following result which subsumes the special case (having finite topological characteristics and finite $\{\partial(q)>1\})$ treated ad hoc in $\S 6$.

Theorem 11.1: If $\Theta$ is of the second kind, then every admitted isolated planar boundary element of $\varphi(\Omega)$ is pointlike and has a deleted neighborhood containing no points of $\varphi(\Omega \cap C)$.

Proof: The case where $C-\Omega$ has at most two members is included in the developments of $\S 6$. It may be treated directly.

In the remaining case $C-\Omega$ is a perfect, totally disconnected set and we introduce $\psi$, a conformal universal covering of $\Omega$ with domain $\Lambda$ 
and group of conformal automorphisms $\Gamma_{0}$. We let $\varphi_{1}=\varphi \circ \psi$ and let $\Gamma_{1}$ denote the group of conformal automorphisms of $\Delta$ which leave $\varphi_{1}$ invariant. For each $\sigma \in \Gamma_{1}$ there exists a unique $\tau_{\sigma} \in \Theta$ such that

$$
\psi \circ \sigma=\tau_{\sigma} \circ \psi \text {. }
$$

We consider an admitted isolated planar boundary element of $\varphi(\Omega)$. With it we associate a univalent conformal map $\alpha$ of a plane annulus $\{1<|z|<r(\leq+\infty)\}$ into $\varphi(\Omega)$ such that $\alpha(z)$ tends to the boundary element in question when $|z|$ tends to $r$ and also $\partial[\alpha(z)]=1,1<|z|<r$. Suppose that $r<+\infty$. It follows from the covering properties of $\varphi_{1}$ that

$$
\alpha\left(e^{z}\right)=\varphi_{1} \circ A(z),
$$

where $A$ is an analytic function on $\{0<\operatorname{Re} z<\log r\}$ taking values in $\Delta$ and having the property that

$$
\lim _{\operatorname{Re} z \rightarrow \log r}|A(z)|=1 .
$$

Hence we conclude by the Schwarz reflexion principle, the univalence of $x\left(e^{z}\right)$ on $\{0<\operatorname{Re} z<\log r ;|\operatorname{Im} z|<\pi\}$, and (11.3) that $\varphi_{1}$ is univalent on $\Delta \cap\left\{\left|z-e^{i \theta}\right|<\varrho\right\}$ for some real $\theta$ and positive $\varrho$. This implies that $\Theta\left(\Gamma_{1}\right)$ is of the second kind and hence that $\Theta\left(\Gamma_{0}\right)$ is of the second kind. Since $C-\Omega$ is totally disconnected, $\Theta\left(\Gamma_{0}\right)$ is of the first kind. The assumption that $r<+\infty$ is untenable. It follows that the admitted isolated planar boundary element under consideration is pointlike.

We next show that $\alpha$ may be so chosen that its image does not intersect $\varphi(\Omega \cap C)$. To that end we make use of the following theorem which we have essentially established elsewhere [5]. It will also be used in Theorem 11.3 .

Theorem 11.2: Given a group $\Theta$, there is a one-to-one map onto the set of admitted pointlike isolated planar boundary elements of $\varphi(\Delta)$ from the set of conjugate classes

$$
K(P)=\left\{\theta P \theta^{-1} \mid \theta \in \Theta\right\},
$$

where $P$ is a maximal cyclic subgroup generated by a parabolic member of $\Theta$, such that the restriction of $\varphi$ to each sufficiently small disk bounded by an oricycle tangent to $C$ at the fixed point of $\gamma$ ( $\neq$ identity) $\in P$ is a conformal universal covering of an arbitrarily small deleted neighborhood of the isolated planar boundary element of $\varphi(\Delta)$ corresponding to $K(P)$.

In order to apply Theorem 11.2 we shall want to make use of the fact that $\varphi_{1}(w)=\varphi_{1}(z)$ only if $w$ is a member of the orbit of $z$ with respect to $\Gamma_{1}$ (cf. $\left.\S 9\right)$. We conclude that there exists a univalent conformal map of $\varphi(\Omega)$ onto the quotient surface associated with $\Gamma_{1}$ such that the rami- 
fication indices associated with $\Gamma_{1}$ and $\Theta$ respectively agree at corresponding points.

Consequently, we may associate by virtue of Theorem 11.2 with an admitted isolated planar boundary element of $\varphi(\Omega)$ a parabolic element $\sigma \in \Gamma_{1}$ such that the restriction of $\varphi_{1}$ to a sufficiently small disk bounded by an oricycle tangent to $C$ at the fixed point of $\sigma$ is a conformal universal covering of some arbitrarily small deleted neighborhood of the isolated planar boundary element under consideration. We proceed by investigating $\tau_{\sigma}$.

Suppose that $\tau_{\sigma}$ is hyperbolic. Considering (11.2) with $\sigma$ replaced by integral powers of $\sigma$ we conclude that $\psi$ possesses two asymptotic paths terminating at the fixed point of $\sigma$ along which $\psi$ tends to two distinct asymptotic values. The theorem of Lindelöf-Gross-Iversen is contradicted. Suppose that $\tau_{\sigma}$ is elliptic or the identity. Since $\tau_{\sigma}$ has a finite order, (11.2) implies that $\psi \circ \sigma^{n}=\psi$ for some positive integer $n$. But then Theorem 11.2 yields the conclusion that the complement of $\Omega$ with respect to the extended plane possesses an isolated point. We have a contradiction, the complement of $\Omega$ being perfect. We conclude that $\tau_{\sigma}$ is parabolic.

We introduce a disk $D(\subset \Delta)$ the frontier of which is an oricycle tangent to $C$ at the fixed point of $\sigma$ such that the restriction of $\varphi_{1}$ to $D$ is a conformal universal covering of $\varphi_{1}(D)$ for which the group of Decktransformationen consists of the $\sigma^{n}$ ( $n$ integer) restricted to $D$. It follows that the restriction of $\psi$ to $D$ is a conformal universal covering of $\psi(D)$ and that its group of Decktransformationen is a subgroup of the group of the preceding sentence. Hence precisely one of the alternatives holds: (1) $\psi$ is univalent on $D,(2) \psi(D)$ is a simply-connected region $G$ less one of its points. Suppose that the latter alternative occurs. Then $G \subset \Omega, C-\Omega$ being perfect. By the covering properties of $\psi, \psi$ is univalent on each component of $\psi^{-1}(G)$ and hence on $D$. The alternative (2) is to be rejected.

The proof of Theorem 11.1 is now readily completed. We may suppose that $D$ is so chosen that the restriction of $\psi$ to $\bar{D} \cap \Delta$ is univalent. Thanks to (11.2) as well as to the facts that $\tau_{\sigma}$ is parabolic and $C-\Omega$ contains more than two elements we conclude that the restriction of $\psi$ to $\bar{D} \cap \Delta$ admits a continuous univalent extension to $\bar{D}$. We infer with the aid of (11.2) that every $D$ has the property that $\psi(D)$ contains a disk $D_{1}$ contained in $\Delta$ or contained in $\{|z|>1\}$, the circumference of which is tangent to $C$ at the fixed point of $\tau_{\sigma}$. Noting that $\varphi_{1}(D) \supset \varphi\left(D_{1}\right)$, we are led to the conclusion that the admitted isolated planar boundary element of $\varphi(\Omega)$ possesses a deleted neighborhood consisting of a subregion of $\varphi(\Delta)$ (resp. $\varphi(\{|z|>1\})$ ) conformally equivalent to a punctured disk. The second assertion of Theorem 11.1 follows. 
In order to establish the complete relation between the admitted isolated planar boundary elements of $\varphi(\Omega)$ on the one hand and the admitted pointlike isolated planar boundary elements of $\varphi(\Delta)$ and of $\varphi(\{|z|>1\})$ on the other hand, we examine the homomorphism $\sigma \rightarrow \tau_{\sigma}$ further. We have Lemma 11.1: Given $\Theta$ of the second kind, $C-\Omega$ being perfect if $\tau(\in \Theta)$ $i s$ parabolic, then there exists $\sigma\left(\in \Gamma_{1}\right)$ parabolic such that (1) $\tau=\tau_{\sigma}$ and (2) for every disk $D(\subset \Delta)$ the circumference of which is tangent to $C$ at the fixed point of $\sigma$ there exists a disk $D_{1}(\subset \Delta)$ the circumference of which is tangent to $C$ at the fixed point of $\tau$ such that $\psi(D) \supset D_{1}$. A corresponding result holds when " $D_{1}(\subset \Delta)$ " is replaced by $" D_{1} \subset\{|z|>1\} . »$

Proof: Let $G$ denote a component of $\psi^{-1}(\Delta)$. Now $\psi$ maps $G$ univalently onto $\Delta$ and $\tau \circ \psi(z)=\psi \circ \sigma_{1}(z), z \in G$, where $\sigma_{1}$ is a conformal automorphism of $G$. Since $\tau \circ \psi=\psi \circ \sigma_{2}, \sigma_{2} \in \Gamma_{1}$, we infer that $\sigma_{1}(z)=\gamma \circ \sigma_{2}(z), \quad \gamma \in \Gamma_{0}, z \in G$. Hence with $\sigma=\gamma \circ \sigma_{2}$ we see that

$$
\tau \circ \psi=\psi \circ \sigma
$$

To see that $\sigma$ is parabolic we proceed as follows. Let $\psi^{*}$ denote the inverse of the restriction of $\psi$ to $G$. We consider an oricycle $K(\subset \Delta)$ tangent to $C$ at the fixed point, $\zeta$, of $\tau$ and note that $\psi^{*}(z)$ tends to a limit as $z$ tends to $\zeta$ along an arc of $K$. This is easily concluded on noting that $\psi$ may be represented as the composition of (1) a conformal universal covering with domain $\Delta$ of the complement of a set consisting of three points of $C-\Omega$ each distinct from $\zeta$ and (2) an analytic function mapping $\Delta$ into itself. (An alternative proof may be based on the fact that $\psi$ is actually of bounded type as a consequence of the theorem of Myrberg-Royden [8] and a theorem of R. Nevanlinna [7, p. 213] together with the Riesz-Nevanlinna theorem [7, p. 209].) As a consequence of the Phragmén-Lindelöf maximum principle [6, p. 76], $\psi_{K}^{*}$, the restriction of $\psi^{*}$ to the bounded region whose frontier is $\bar{K}$, admits a univalent continuous extension to the bounded closed disk the frontier of which is $\bar{K}$. Since $K$ is mapped onto itself by $\tau$, we infer that $\lim _{|n| \rightarrow \infty} \sigma^{n}(0)=\lim _{\zeta} \psi_{K}$, and hence that $\sigma$ is parabolic.

The remaining points of the lemma are now easily established. The exterior case is similarly treated, " $\Delta$ » being replaced in the first two sentences of the proof by $\|\{|z|>1\} »$.

We now establish

Theorem 11.3: Let there be given $\Theta$ of the second kind. Let $G$ denote the Riemann surface obtained by adjoining to $\varphi(\Omega)$ the admitted isolated planar boundary elements and let $G_{1}$ and $G_{2}$ denote the Riemann surfaces obtained by adjoining to $\varphi(\Delta)$ and $\varphi(\{|z|>1\})$ respectively their admitted pointlike isolated planar boundary elements. Then the identity map of $\varphi(\Delta) \cup \varphi(\{|z|>1\})$ 
admits a unique extension as a conformal map of $G_{1} \cup G_{2}$ into $G$. This extension maps the set

$$
G_{1} \cup G_{2}-[\varphi(\Delta) \cup \varphi(\{|z|>1\})]
$$

onto $G-\varphi(\Omega)$.

Proof: If $C-\Omega$ reduces to at most two elements, the theorem is readily established by direct elementary examination. We put this case aside and continue assuming that $C-\Omega$ has more than two elements. Given $p \in G_{1}-\varphi(\Delta)$, let $\gamma$ be a generating parabolic transformation of $P$ where $p$ corresponds to $K(P)$ in the sense of Theorem 11.2. Let $\sigma\left(\in \Gamma_{1}\right)$ be chosen to satisfy the conditions stated in the first sentence of Lemma 11.1 relative to $\gamma$. It follows from (2) of Lemma 11.1 that the identity map of $\varphi(\Delta)$ into $\varphi(\Omega)$ possesses as a limit at $p$, the boundary element of $\varphi(\Omega)$ corresponding to the admitted pointlike isolated planar boundary element of the quotient Riemann surface relative to $\Gamma_{1}$ associated with $\sigma$ (by virtue of the map carrying the orbit of a point $z$ relative to $\Gamma_{1}$ into $\left.\varphi_{1}(z)\right)$. The proof of the existence and univalence of the extension of the identity map is now readily established. The unicity is, of course, trivial. The onto property is seen to follow from the sentence preceding the last of the proof of Theorem 11.1.

As a consequence of Theorem 11.3 it will be seen that in the study of the relation connecting admitted isolated planar boundary elements of $\varphi(\Omega)$ and the behavior of a fundamental polygon it suffices to control the situation for $\varphi(\Delta)$. 12. Our next concern will be with cusps of a fundamental polygon and their relation to the parabolic transformations of $\Theta$. Before proceeding further, it is desirable to agree upon some formal definitions.

By a cusp of a fundamental polygon $\Pi$ is meant an ordered pair $\left(\gamma_{1}, \gamma_{2}\right)$ of distinct sides of $\Pi$ lying in $\Delta$ and having a common endpoint, $\zeta$, on $C$ such that the order at an interior point of $\Pi$ is one for a periodic parametric representation, $Z$, of the closed Jordan curve fr $\Pi$, where $Z$ is so chosen that points of $\gamma_{1}$ precede and points of $\gamma_{2}$ follow $\zeta$ locally for parameter values in a sufficiently small neighborhood of an antecedent of $\zeta$. (To be specific, $Z$ is a continuous map of the real line onto fr $\Pi$ with period one which is univalent on $\{0 \leq t<1\}$.) We term $\zeta$ the vertex of the cusp. An ordered pair $\left(k_{1}, k_{2}\right)$ of cusps of $\Pi$ will be termed allowable provided that there exists $\tau(\in \Theta)$, mapping some subarc of the second component of $k_{1}$ one of the endpoints of which is the vertex of $k_{1}$ onto a subarc of corresponding kind of the first component of $k_{2}$ and mapping the vertex of $k_{1}$ onto the vertex of $k_{2}$. There is at most one such $\tau$. Given $k_{1}$, there is at most one $k_{2}$ such that $\left(k_{1}, k_{2}\right)$ is allowable. Given a cusp $k$, there exists a unique maximal sequence $\left(k_{n}\right)_{l}^{m}$ 
of cusps $k_{n},-\infty \leq l \leq 0 \leq m \leq+\infty$, such that $k_{0}=k$ and that for each finite $j, l \leq j<j+1 \leq m$, the ordered pair $\left(k_{j}, k_{j+1}\right)$ is allowable. By a periodic cusp sequence is meant a sequence of the above type such that for some positive integer $p$ we have $k_{n+p}=k_{n}$. We define cusp sequences $\left(k_{n}\right)$ and $\left(k_{n}^{\prime}\right)$ to be equivalent provided that the set of cusps of each is the same. It need hardly be remarked that a bona fide equivalence relation is so defined and that if $k_{m}^{\prime}=k_{m+v}, m$ and $v$ integers, then $k_{n}^{\prime}=k_{n+v}$ for all $n$ for which $k_{n}^{\prime}$ is defined and the two sequences are equivalent. We now have the prerequisite terminology for the following theorem.

Theorem 12.1: Given $\Theta$, let a fundamental polygon $\Pi$ be fixed and let cusps be taken to mean cusps of $\Pi$. There exists a univalent map of the admitted pointlike isolated planar boundary elements of $\varphi(\Delta)$ onto the set of equivalence classes of periodic cusp sequences which is such that $\varphi(z)$ tends to a given admitted pointlike isolated planar boundary element when $z$ tends within $\Pi$ to a vertex of a cusp of a periodic cusp sequence belonging to the equivalence class onto which the given boundary element of $\varphi(\Delta)$ is mapped. There is but one such map. There is a unique univalent map from the set of periodic cusp sequences onto the set of $K(P)$ such that the vertex of each cusp of a periodic cusp sequence is a fixed point of the elements of a group in the image class.

Proof: We consider the extension $G_{1}$ of $\varphi(\Delta)$ introduced in Theorem 11.3 (however $\Theta$ is now unrestricted) and introduce a uniformizer $\theta$ with domain $\Delta$ such that $\theta(0)$ is a given point $p \in G_{1}-\varphi(\Delta)$ and $\theta(z) \in \varphi(\Delta)$ and $\partial[\theta(z)]=1,0<|z|<1$. Let $D=\theta(\{0<|z|<1 / 2\})$. We investigate the subset of $\Delta \cap$ fr $\Pi$ mapped by $\varphi$ into $D$.

We first show that if $K$ is a component of $\Delta \cap \mathrm{fr} \Pi, \varphi(K)$ - $\bar{D}$. Suppose, on the contrary, that there exists a component $K_{0}, \varphi\left(K_{0}\right) \subset \bar{D}$. We are assured that $\varphi(z)$ tends to $p$ as $z$ tends along $K_{0}$ to one or the other of its endpoints. Two alternatives present themselves. The first is that the restriction of $\varphi$ to $K_{0}$ is univalent. But then the closed Jordan curve $\overline{\varphi\left(K_{0}\right)}$ would separate $\varphi($ int $\Pi)$, which is connected. The second alternative is that the restriction of $\varphi$ to $K_{0}$ is not univalent. In this case there would exist a closed subarc of $K_{0}$, say $\gamma$, such that $\varphi(\gamma)$ is a closed Jordan curve since by the choice of $\theta$ at each point of $K_{0}$ the multiplicity of $\varphi$ is one. This circumstance is excluded by the reason used in the case of the first alternative. We conclude that $\varphi(K) c_{-} \bar{D}$ for each component $K$ of $\Delta \cap$ fr $\Pi$.

We next consider the set of $K$ for which $\varphi(K) \cap D \neq \varnothing$. For each such $K, \varphi(K)$ intersects $\theta(\{|z|=1 / 2\})$. Since some neighborhood of a point of $\varphi(\Delta)$ contains points of $\varphi(K)$ for only a finite set of $K$, the set of $K$ for which $\varphi(K) \cap D \neq \varnothing$ is finite. 
For a given $K$ such that $\varphi(K) \cap D \neq \varnothing$, let $\gamma$ denote the smallest connected subset of $K$ containing the points of $K$ mapped by $\varphi$ into $\theta(\{|z|=1 / 2\})$. A component of $\varphi^{-1}(D) \cap K$ either lies in $\gamma$ or else is one of the components of $K-\gamma$. Since each $\gamma$ is compact, there is a neighborhood of $p$ having no points in common with any $\varphi(\gamma)$. If a component of $K-\gamma$ is a component of $\varphi^{-1}(D) \cap K$, then $\varphi(z)$ tends to $p$ as $z$ tends on such a component to its endpoint on $C$. We term such a component an $\alpha$-component.

$\alpha$-components exist. For if this were not the case, some deleted neighborhood of $p$ would lie in $\varphi($ int $\Pi$ ), and consequently the inverse of the restriction of $\varphi$ to int $\Pi$ would possess a limit at $p$ which lies in int $\Pi$. This is not possible.

The reasoning used in showing that $\varphi(K) c_{-} \bar{D}$ when $\varphi$ is not univalent on $K$ may be applied to show that $\varphi$ is univalent on an $x$-component.

Suppose that there are given $\alpha$-components of $\varphi^{-1}(D) \cap K_{j}, j=1,2$, which have intersecting images with respect to $\varphi$. A renewed application of the reasoning just referred to yields the result that the $\varphi$-images of these $\alpha$-components have in common a subarc one of the endpoints of which is $p$. It suffices to note that otherwise there would exist a closed Jordan curve in $\bar{D}$ formed by taking subarcs of the images with respect to $\varphi$ of the $\alpha$-components which would be disjoint but have the same endpoints and thereupon introducing the closure of their union.

If $\alpha_{1}$ is a given $\alpha$-component, $\varphi\left(\alpha_{1}\right)$ contains points arbitrarily near $p$ and hence points not in any $\varphi(\gamma)$. Since a point of $\varphi\left(\alpha_{1}\right)$ is accounted for at least at two distinct points on $\Delta \cap \mathrm{fr} \Pi$, it follows that there exists a second $\alpha$-component, $\alpha_{2}$, distinct from $\alpha_{1}$, such that $\varphi\left(x_{1}\right)$ and $\varphi\left(\alpha_{2}\right)$ have in common a subarc with endpoint $p$. We are led to the following conclusion of fundamental importance for the further developments:

There exists an open Jordan disk $D_{1}, p \in D_{1} \subset D$, such that

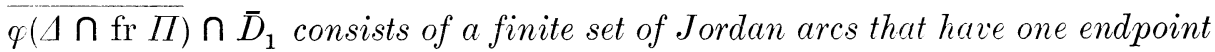
on fr $D_{1}$ and the other at $p$, that two distinct arcs have only $p$ in common, and that each arc is a subarc of the $\phi$-image of some $x$-component.

The antecedent of a component of $D_{1}$ less the arcs in question with respect to the restriction of $\varphi$ to int $\Pi$ is a Jordan region the frontier of which contains subarcs of two distinct $x$-components whose closures have in common exactly one point on $C$. These subares lie on sides of $\Pi$ which are the components of a cusp, the possibility of a corner of fr $\Pi$ on these subarcs being excluded. Further two distinct components of $D_{1}-\overline{\varphi(\Delta \cap \mathrm{fr} \Pi)}$ which abut on one of the component arcs of $\varphi(\Delta \cap \mathrm{fr} \bar{\Pi}) \cap D_{1}$ lead to two cusps (obtained in the manner just described) which are the components of an allowable ordered pair of cusps. It may now be concluded that a 
cusp so associated with a component of $D_{1}-\overline{\varphi(\Delta \cap \mathrm{fr} \Pi)}$ is $k_{0}$ for some periodic cusp sequence, $\left(k_{n}\right)_{-\infty}^{+\infty}$, having the property that $\varphi(z)$ tends to $p$ when $z$ tends to the vertex of $k_{n}$ ( $n$ arbitrary integer $)$ in $\Pi$. If a second periodic cusp sequence, $\left(k_{n}^{\prime}\right)_{-\infty}^{+\infty}$, also has this property, it must be equivalent to $\left(k_{n}\right)_{-\infty}^{+\infty}$. This may be seen as follows. We first note that the image with respect to $\varphi$ of a sufficiently small subarc of a component of $k_{n}^{\prime}$ having the vertex of $k_{n}^{\prime}$ as an endpoint is a subarc with endpoint $p$ of a component of $\varphi(\Delta \cap \mathrm{fr} \Pi) \cap D_{1}$. If the images with respect to $\varphi$ of small subarcs of the components of $k_{n}^{\prime}$ having the vertex of $k_{n}^{\prime}$ as endpoint have a point $(\neq p)$ in common, then $\varphi(\Delta \cap \mathrm{fr} \Pi) \cap D_{1}$ has one component and $k_{n}^{\prime}=k_{m}$, all $n$. If, however, they are disjoint, they lie on the frontier of a component of $D_{1}-\overline{\varphi(\Delta \cap \text { fr } \Pi)}$ and $k_{n}^{\prime}=k_{m}$, some $m$ and the "period» exceeds one. We conclude readily the second and third sentences of Theorem 12.1 with the sole reservation that the map in question has not yet been shown to be »onto». The univalence is, of course, obvious.

"Onto». Given the periodic cusp sequence $\left(k_{n}\right)_{-\infty}^{+\infty}$, let $v$ denote the smallest positive integer such that $k_{n+v}=k_{n}$. We fix an integer $m$ and let

$$
\tau=T_{1}^{-1} T_{2}^{-1} \ldots T_{v}^{-1}
$$

where $T_{j}$ is the member of $\Theta$ mapping points of the second component of $k_{m+j-1}$ near the vertex of $k_{m+j-1}$ into points of the first component of $k_{m+j}$ near the vertex of $k_{m+j}$. We see that $\tau \neq$ identity and that $\zeta$, the vertex of $k_{m}$, is a fixed point of $\tau$. Hence $\tau$ is either hyperbolic or parabolic. The former possibility will now be excluded.

Suppose $\tau$ hyperbolic and let $\eta$ denote the other fixed point of $\tau$. The non-euclidean straight line with endpoints $\zeta$ and $\eta$ cannot contain points of $\Pi$ arbitrarily near $\zeta$. Otherwise, there would exist an orbit having infinitely many members in $\Pi$. To continue, we note that the bounded region, $\omega$, the frontier of which consists of the sides of the cusp $k_{m}$ together with their endpoints and the non-euclidean straight line (resp. ray, segment) joining the endpoints different from the vertex has no points in common with the non-euclidean straight line joining $\zeta$ and $\eta$. Since $\tau$ is taken to be hyperbolic, every neighborhood of a given point of the non-euclidean straight line with endpoints $\zeta$ and $\eta$ intersects $\tau^{n}(\omega)$ for infinitely many integers $n$. This is impossible, for a sufficiently small neighborhood of a point of $\Delta$ intersects $\sigma(\Pi)$, and a fortiori $\sigma(\omega)$, for only a finite set of $\sigma \in \Theta$. We conclude that $\tau$ is parabolic.

There exists a circular disk $\Delta_{1} \subset \Delta$ the circumference of which is tangent to $C$ at the fixed point of $\tau$ such that if two points of $\Delta_{1}$ are in the same orbit with respect to $\Theta$, they are in the same orbit with respect to the 
group $P$, generated by $\tau$. Since the image of $\Delta_{1}$ with respect to the orbit map associated with $P$ is conformally equivalent to a punctured disk, it follows that $\varphi\left(\Delta_{1}\right)$ is also conformally equivalent to a punctured disk and is a deleted neighborhood of a point of $G_{1}-\varphi(\Delta)$. It is easily verified that $\left(k_{n}\right)_{-\infty}^{+\infty}$ stands in the stated relation to this point.

(It is clear that the »empty» case: $G_{1}-\varphi(\Delta)=\varnothing$, is cared for by the above argument.)

The final sentence of the theorem is now readily established with the aid of Theorem 11.2 and the fact that the vertex of each cusp of a periodic cusp sequence is the fixed point of a parabolic member of $\Theta$.

Several supplementary remarks are in order. First, when $\Theta$ of the second kind is considered, the totality of admitted isolated planar boundary elements of $\varphi(\Omega)$ is accounted for by the periodic cusp sequences and the corresponding entities that arise from considering the reflexion of $\Pi$ with respect to $C$.

Second, we note that in the case where $\Theta$ is Fuchsian, a cusp the vertex of which lies on $C-\Omega$ is a term of a periodic cusp sequence. For a transformation $\tau$ ( $\neq$ identity) of $\Theta$ which maps a component of a cusp into a side of $\Pi$ carries the vertex of the cusp into a point of $C-\Omega$ which is, as a point of $C-\Omega$, the vertex of a cusp of $\Pi$. Our assertion follows from the finitary properties of $\Pi$.

Finally, we make a remark concerning the nature of the image with respect to $\varphi$ of a component side of a cusp that is a term of a periodic cusp sequence. Suppose that $\gamma$ is a sufficiently small subarc of a side of a cusp with one endpoint the vertex. We shall show that $\overline{\varphi(\gamma)}$ is a regular analytic Jordan arc on $G_{1}$. This may be concluded from the observation that the restriction of $\varphi$ to a sufficiently small disk $\Delta_{1}(\subset \Delta)$ the circumference of which is tangent to $C$ at a fixed point $\zeta$ of a parabolic transformation of $\Theta$ may be represented as $\Phi \circ \psi$, where $\psi$ is a conformal universal covering of $\Delta-\{0\}$ which maps the maximal open arcs of the hyperbolic straight lines which lie in $\Delta_{1}$ and have $\zeta$ as an endpoint onto the open radii of $\Delta$ and $\Phi$ is a univalent conformal map of $\Delta$ into $G_{1}$.

The following question appears to be fairly recondite: In the case of Poincaré polygons what is the dependence of the vertices of the cusps of the periodic cusp sequence on the base point $a$ ? Since the vertices constitute a countable set, namely the set of the fixed points of the parabolic transformations of $\Theta$, the problem of the dependence of the periodic cusp sequences associated with a given point of $G_{1}-\varphi(\Delta)$ on $a$ is presumably complicated, especially in the Fuchsoid case.

13. We have just seen that in the case of a Fuchsian group a vertex of a cusp of $\Pi$ which lies on $C-\Omega$ is necessarily the fixed point of some 
parabolic member of the group. The situation changes radically in the case of a Fuchsoid group as the following examples show.

Let us introduce the hyperbolic straight lines $\lambda_{k}$ with endpoints $\exp \left(\pi i \sum_{0}^{k-1} 2^{-j}\right)$ and $\exp \left(\pi i \sum_{0}^{k} 2^{-j}\right), k$ a positive integer. Let $\lambda_{-1}$ denote the hyperbolic straight line with endpoints 1 and $i$ and let $\lambda_{0}$ denote the hyperbolic straight line with endpoints $i$ and -1 . Let $\tau_{k}$ denote the elliptic Möbius transformation preserving $\Delta$ which is of period two and has as a fixed point the point of $\lambda_{k}$ equidistant in the euclidean sense from the endpoints of $\lambda_{k}$. The group generated by the $\tau_{k}$ is a $\Theta$ and the associated $\Pi(0)$ has as frontier the closure of the union of the $\lambda_{k}$. It is readily verified that no cusp of $\Pi(0)$ is a term of a periodic cusp sequence. The group so formed is of the first kind. The associated cusp sequence is univalent and infinite on one side. A group of the second kind having similar properties is obtained when $\lambda_{k}$ is replaced by the hyperbolic straight line having endpoints $\exp \left(\frac{\pi i}{2} \sum_{0}^{k-1} 2^{-j}\right)$ and $\exp \left(\frac{\pi i}{2} \sum_{0}^{k} 2^{-j}\right), k=1,2, \ldots$ and only such $k$ are considered. Examples of groups of either kind where cusp sequences that are univalent and infinite on both sides are present are now readily constructed.

14. The following theorem appears as an easy consequence of Siegel's theorem together with Theorem 11.2. (The second part of the conclusion is perhaps known, granted $\Theta$ Fuchsian.)

Theorem 14.1: Let there be given a group $\Theta$ and $\Theta_{1}$ a subgroup of $\Theta$. Suppose that $\Theta_{1}$ is a Fuchsian group of the first kind. Then $\Theta$ is also a Fuchsian group of the first kind and there exists a positive integer $m$ such that $\tau^{m} \in \Theta_{1}$ for every $\tau \in \Theta$.

Proof: Since $\Theta_{1}$ is a Fuchsian group of the first kind, the hyperbolic area of $\Pi(a)$ (belonging to $\Theta_{1}$ ) is finite. The Poincaré polygon based at $a$ for $\Theta$ being contained in $\Pi(a)$ has finite hyperbolic area. Consequently, we conclude by Siegel's theorem that $\Theta$ is Fuchsian.

Now let $\varphi_{1}$ denote the quotient map associated with $\Theta_{1}, \varphi_{1}(z)$ being the orbit of $z$ with respect to $\Theta_{1}$. We have: $\varphi=\psi \circ \varphi_{1}$, where $\psi$ is a conformal map of $\varphi_{1}(\Delta)$ onto $\varphi(\Delta)$. The situation is familiar when $\varphi_{1}(\Delta)$ is compact, $\psi$ being a map of constant valence of $\varphi_{1}(\Delta)$ onto $\varphi(\Delta)$ and the polygons for the two groups being compact. Suppose then that $\varphi_{1}(\Delta)$ is not compact. In this case it is conformally equivalent to a compact Riemann surface less a finite set of points. Recalling Theorem 11.2 we see that a circular disk $\Delta_{1}(\subset \Delta)$ the circumference of which is tangent to $C$ at the fixed point of a parabolic transformation $\sigma \in \Theta_{1}$ and which has sufficiently small radius has the property that $\varphi_{1}\left(\Delta_{1}\right)$ and $\varphi\left(\Delta_{1}\right)$ are arbitrarily 
small deleted neighborhoods of boundary elements of $\varphi_{1}(\Delta)$ and $\varphi(\Delta)$ respectively. It follows that $\psi(p)$ tends to the ideal boundary of $\varphi(\Delta)$ when $p$ tends to the ideal boundary of $\varphi_{1}(\Delta)$. We conclude that the valence of $\psi$ is finite and constant.

Let $q(\in \varphi(\Delta))$ be such that $\partial(q)=1$, let $\varphi(z)=q$, and let $v$ denote the constant value of the valence of $\psi$. Given $\tau \in \Theta$, there exist integers $\mu_{1}$ and $\mu_{2}, \quad 1 \leq \mu_{1}<\mu_{2} \leq v+1 \quad$ such that $\varphi_{1}\left[\tau^{\mu_{1}}(z)\right]=\varphi_{1}\left[\tau^{\mu_{2}}(z)\right]$. Hence $\tau^{\mu_{2}-\mu_{1}}\left[\tau^{\mu_{1}}(z)\right]=\sigma\left[\tau^{\mu_{1}}(z)\right], \sigma \in \Theta_{1}$. We conclude that $\sigma=\tau^{\mu_{2}-\mu_{1}}$, and the theorem follows. Actually slightly more has been established since for each $\tau \in \Theta$ we are assured that for some positive integer $\mu, 1 \leq \mu \leq v$, $\tau^{\mu} \in \Theta_{1}$.

The same argument also shows that if $\tau_{1}, \ldots, \tau_{v} \in \Theta$, then there exist $\mu_{1}, \mu_{2}, 1 \leq \mu_{1} \leq \mu_{2} \leq v$, such that

$$
\stackrel{\mu_{2}}{\prod_{\mu_{1}}} \tau_{\lambda} \in \Theta_{1}
$$

\section{Bibliography}

[1] Ahlfors, L. V. An extension of Schwarz's Lemma. Trans. A.M.S. 43 (1938), $359-364$.

[2] Appell, P. and Goursat, É. Théorie des fonctions algébriques d'une variable et des transcendantes qui s'y attachent. vol. 2. (Fonctions automorphes, P. Fatou). Paris 1930.

[3] Heins, M. On a class of conformal metrics. Nagoya Journal of Mathematics 21 (1962), $1-60$.

[4] -》- A class of conformal metrics. Bull. A.M.S. 67 (1961), 475-478. (A summary of $[3])$.

[5] - - On Fuchsoid groups that contain parabolic transformations. Contributions to Function Theory. Tata Institute of Fundamental Research, Bombay (1960), 203-210.

[6] -»- Selected Topics in the Classical Theory of Functions of a Complex Variable. New York 1962.

[7] Nevanlinna, R. Eindeutige analytische Functionen 2d. ed. Berlin, Heidelberg 1953.

[8] Royden, H. L. Harmonic functions on open Riemann surfaces. Trans. A.M.S. 73 (1952), 40-94.

[9] Siegel, C. L. Some remarks on discontinuous groups. Ann. of Math. (2) 46 (1945), $708-718$.

[10] -»- Ausgewählte Fragen der Funktionentheorie II. Göttingen, 1954.

[11] Tsus, M. Potential Theory in Modern Function Theory. Tokyo, 1959.

University of Illinois. 